\title{
Thrombolysis and thrombectomy for acute ischaemic stroke
}

\author{
Authors: Salwa El Tawil ${ }^{\mathrm{A}}$ and Keith W Muir ${ }^{\mathrm{B}}$
}

The likelihood of disability-free recovery after acute ischemic stroke is significantly improved by reperfusion either by intravenous thrombolytic drug treatment or with endovascular mechanical thrombectomy in selected cases. The use of intravenous thrombolysis is limited by the short treatment window and you need to assess individual balance of benefit and risk of symptomatic intracranial haemorrhage. Benefit is greater for shorter onset-to-reperfusion time intervals, requiring optimisation of pre-hospital and inhospital pathways. Symptomatic haemorrhage is more likely with more severe strokes, but a greater proportion of patients are left free of disability than suffer a treatmentrelated haemorrhage at all levels of severity. Extracranial haemorrhage and orolingual angioedema are less common complications. Endovascular mechanical thrombectomy can be used in selected patients with imaging-proven large artery occlusion. Successful therapy depends on well-organised services that can deliver treatment within a short time window at centres with adequate expertise to perform the procedure.

\section{Introduction}

Brain tissue may be rescued if blood flow is restored rapidly after onset of acute ischemic stroke. Intravenous recombinant tissue plasminogen activator (IV rtPA) and, since 2015, endovascular thrombectomy (intra-arterial therapy, IAT) using stent retrievers can significantly improve the odds of disabilityfree recovery.

\section{Intravenous thrombolysis}

Pooled individual patient data meta-analysis of 6,756 patients in nine randomised controlled trials (RCTs) of IV rtPA show significantly increased likelihood of recovery to independence with treatment initiated up to 4.5 hours after symptom onset. The likelihood of favourable outcome declines rapidly with longer onset-to-treatment times: the number needed to treat (NNT) for excellent functional outcome is five for treatment within 90 minutes, nine for

Authors: ${ }^{\text {A }}$ clinical research fellow, Institute of Neuroscience and Psychology, University of Glasgow, Glasgow, UK; ${ }^{\text {BSINAPSE }}$ professor of clinical imaging and consultant neurologist, Institute of Neuroscience and Psychology, University of Glasgow, Glasgow, UK treatment within 91-180 minutes, and 19 for treatment within 181-270 minutes after onset. ${ }^{1}$

Clinical uptake of IV rtPA remains limited by a clinically challenging diagnosis, short therapeutic time window and the need to balance risk and benefit in individuals, taking into account relative contraindications. ${ }^{2}$ Service organisation to optimise pre- and in-hospital pathways can, however, result in $20 \%$ of patients being eligible using current guidelines. ${ }^{3}$ While there are some clear contraindications, many 'legacy' contraindications arising from older clinical trials and reflected in the drug label have been superseded in light of evidence of equivalent safety and efficacy. ${ }^{4}$ There is net benefit for IV rtPA across all strata of severity and age with respect to reduction in disability, the dominant outcome after stroke (suggested framework in Fig 1).

\section{Key points}

Intravenous recombinant tissue plasminogen activator (IV rtPA) given within 4.5 hours of symptom onset significantly increases the likelihood of recovery to independence

Greater benefit is seen with shorter onset-to-treatment times and services should minimise delay in instituting treatment

Symptomatic intracranial bleeding is the most severe possible complication of IV rtPA; other complications include systemic bleeding and orolingual oedema

The treatment decision depends on balancing the possibility of good clinical outcome against the risk of intracranial bleeding

Endovascular mechanical thrombectomy improves the chances of good outcome in a subset of patients with large vessel occlusion

Complications of endovascular procedures include those related to vascular access, radiological contrast media and devicerelated vascular injury

KEYWORDS: Cerebrovascular disease, endovascular treatment, stroke, thrombectomy, thrombolysis 
Patient with sudden onset of focal neurological deficit

Indications for IV rtPA:

- clinical picture consistent with acute stroke

- last seen well less than 4.5 hours ago

YES

Absolute contraindications for IV rtPA:

- arterial blood pressure more than 185 systolic or 110 diastolic despite treatment

- high risk of bleeding

- INR>1.6

- PT>15

- platelet count $<100,000$

- hereditary or acquired bleeding disorders

- use of therapeutic doses of IV or oral anticoagulants within the last 48 hours

- extensive hypodensity on initial brain CT

- evidence of intracranial haemorrhage on initial brain CT

- recent extensive trauma (including surgery, arterial or lumbar puncture)

- high risk of intracranial pathology, eg glioblastoma

\section{NO}

Relative contraindications for IV rtPA

- NIHSS $>25$

- blood sugar $<2 \mathrm{mmol} / \mathrm{L}$ or $>18 \mathrm{mmol} / \mathrm{L}$ with persistence of symptoms on treatment

- recent minor trauma or surgery

- low risk intracranial structural lesions, eg meningioma, small

asymptomatic aneurysm

- seizure at onset

- previous intracranial haemorrhage

- pregnancy or postpartum

- recent myocardial infarction ratio; IV rtPA = intravenous recombinan tissue plasminogen activator; NIHSS = National Institutes of Health Stroke Scale: $\mathrm{PT}=$ prothrombin time
Thrombolysis
NO

No thrombolysis
YES

No thrombolysis
YES

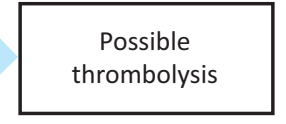
risk benefit

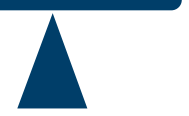

\section{Indications and contraindications}

Treatment decisions should take into account individual riskbenefit balance and may be informed by modelled RCT data illustrating outcomes in different strata of severity and onset-totreatment time. ${ }^{5}$ While the relative benefit of treatment remains constant across age groups and severities, the absolute benefit varies by time to treatment and should be balanced against the risk of symptomatic intracerebral haemorrhage (SICH). Relative risk of SICH appears constant across the first 4.5 hours. Since risk of SICH increases with stroke severity, and with the presence of pre-existing brain imaging abnormalities such as old ischaemia, absolute excess SICH risk increases with increasing stroke severity. However, risk factors for SICH are also those that predict poor outcome from stroke without treatment (eg advanced age, high blood glucose, more severe stroke, pre-existing computerised tomography (CT) changes) and treatment net benefit is consistent across all of these subgroups.

Effects in very mild stroke (National Institutes of Health Stroke Scale (NIHSS) score $\leq 5$ ) are imprecisely characterised, prompting further clinical trials in this situation. ${ }^{6}$ Clinical judgement should be preferred to neurological scores: very significant deficits may be associated with low NIHSS scores (for example, complete hemianopia or isolated dysphasia), while very high NIHSS scores $(>25)$ are predominantly seen in posterior circulation stroke. ${ }^{7}$

Brain imaging is essential to exclude intracranial haemorrhage and established recent ischaemia. Other structural intracranial pathologies were excluded from clinical trials largely to ensure 
correct diagnosis and, therefore, there are very limited data on safety or efficacy in patients with other structural lesions. The presence of anatomically extensive hypodensity is a marker of extensive irreversible ischaemia (a 'large ischaemic core') and signifies a worse prognosis although the relative treatment effect of IV rtPA does not differ in this group. ${ }^{8,9}$ Multimodal imaging, including CT or magnetic resonance angiography and MRI or CT perfusion (CTP) imaging, yields information on intracranial vessels and brain perfusion, and can differentiate between ischaemic core and potentially reversible ischaemia (the penumbra). ${ }^{10}$ While imaging features, especially large ischaemic core volumes and poor collateral vessel status, are strongly linked to prognosis, ${ }^{11}$ an interaction with treatment effect is not established. ${ }^{12}$

Treatment is contraindicated in conditions where there is considered to be a high risk of intracranial or systematic haemorrhage. ${ }^{13}$ These include coagulopathies and elevated blood pressure $(>185 \mathrm{mmHg}$ systolic or $>110 \mathrm{mmHg}$ diastolic by licence). Data from registries, case-control studies and large case series show thrombolysis to be of similar safety and effectiveness in a range of clinical situations previously considered relative contraindications, including history of previous stroke and diabetes, stroke due to dissection, recent stroke ( $>6$ weeks ago), minor silent subacute stroke, hypertension within the range $<185 / 110 \mathrm{mmHg}$, increased international normalised ratio $<1.7$, myocardial infarction more than 7 weeks earlier, asymptomatic intracranial aneurysms, presence of early ischaemic change on CT, leukoaraiosis, MRI-defined cerebral microbleeds and very severe stroke. The third International Stroke Trial (IST-3) included patients with a broader range of baseline characteristics than earlier trials undertaken to establish efficacy and found no significant modification of treatment effect by a range of characteristics, including early ischaemic change on CT, presence of leukoaraiosis, baseline blood pressure (including some patients with elevated blood pressure beyond licence guidelines), blood glucose, age, stroke severity, prior antiplatelet therapy use, history of diabetes or atrial fibrillation. ${ }^{9}$

\section{Complications}

Minor systemic bleeding is common at sites of trauma (eg venepuncture sites, gums, oropharynx and minor injuries). $\mathrm{SICH}$ is the complication most likely to have functional importance. The widely used European Cooperative Acute Stroke Study radiological classification of ICH (Fig 2) is combined with clinical change in various ways across study reports and rates of SICH range from $2-6 \%$ depending on the definition. Only parenchymal haematoma type 2 bleeds have been associated independently with poor outcome, while haemorrhagic infarction is associated with reperfusion. ${ }^{11,14}$ Several factors increase SICH risk, ${ }^{15,16}$ but stroke severity is the strongest predictor (with or without thrombolysis). ${ }^{17}$ Excess risk of SICH rises from 1.5\% for NIHSS 0-4 to 3.7\% for NIHSS $>25$ (Fig 2). There is no evidence to support procoagulant therapy or surgical intervention for SICH.

Rates of extra-cranial haemorrhage after IV rtPA varied from $2-7 \%$, but no consistent definition has been used and treatment has been dictated by site and volume of bleeding. ${ }^{18}$

Orolingual angioedema, an idiosyncratic acute swelling of the lips and tongue, is associated with prior angiotensin converting enzyme inhibitor therapy and occurs in $1-8 \%$ of patients

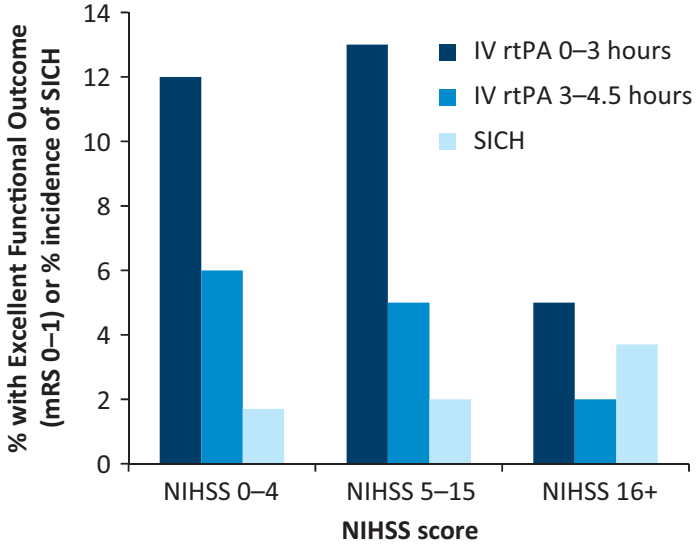

Fig 2. Risk of symptomatic intracerebral haemorrhage (SICH) and absolute increase in proportion of patients with excellent functional outcome at 3-6 months after stroke by stroke severity (National Institutes of Health Stroke Scale (NIHSS)). These represent the difference in absolute outcomes between intravenous recombinant tissue plasminogen activator (IV rtPA)-treated patients and control groups adjusted for age and treatment delay. Modelled excess SICH risks for NIHSS strata were adjusted for age and treatment delay. Data derived from Whiteley et al. ${ }^{5}$

receiving IV rtPA. Cases may be unilateral or bilateral, and occur 15 minutes to a few hours after therapy. Approximately $70 \%$ of cases are classed as mild, with $12 \%$ sufficiently severe to compromise breathing. Most cases resolve spontaneously or with the use of corticosteroids or antihistamines. ${ }^{19}$ Other allergic reactions to IV rtPA are rarely reported. ${ }^{20}$

Neurological deterioration after IV rtPA is more than twice as likely to be caused by non-haemorrhagic factors (recurrent ischaemic stroke, brain swelling or non-attributable deterioration) than SICH. ${ }^{21}$ Systemic illness, including infection, hypoxia or other reversible causes, may all worsen a patient's neurological condition and should be sought.

\section{Mechanical endovascular thrombectomy}

Early recanalisation after IV rtPA is infrequent in occlusions of large intracranial vessels ( $<10 \%$ of terminal internal carotid artery or $25 \%$ of middle cerebral artery M1 segment $)^{22}$ and where there is a large clot burden. Occlusions of these sites are therefore associated with very severe stroke and poor outcome, but likelihood of early recanalisation is lowest with IV rtPA. Endovascular mechanical thrombectomy was shown to be effective in this group of patients in five RCTs published in 2015 (and confirmed in three others), with significantly improved odds of favourable outcome compared with best medical care alone (including IV thrombolysis in $90 \%$ of randomised patients). It has an NNT of 8 to achieve excellent clinical outcome and an NNT of 3 to move to a more favourable functional outcome. There was no increase in mortality or SICH incidence. ${ }^{23}$ All positive trials used stent-retriever devices, which may achieve recanalisation in up to $87 \%$ of patients.

All trials selected patients with large artery occlusion using CT angiography. In three trials, additional selection to enrich the population using perfusion or vascular collateral imaging was undertaken. Centres provided highly organised, high-volume 


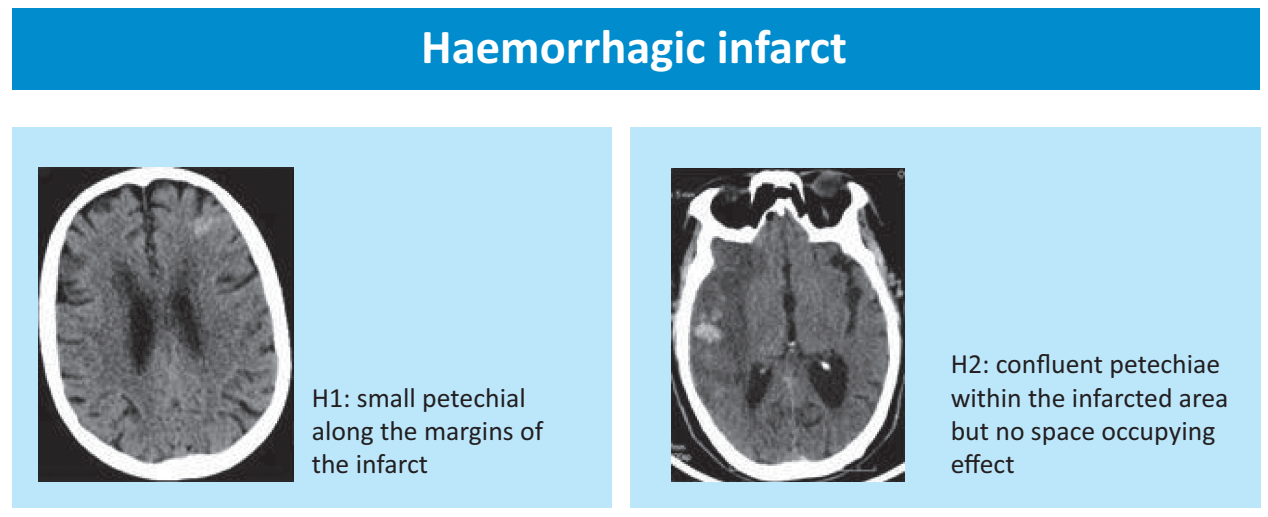

Fig 3. Classification of postthrombolysis haemorrhage.
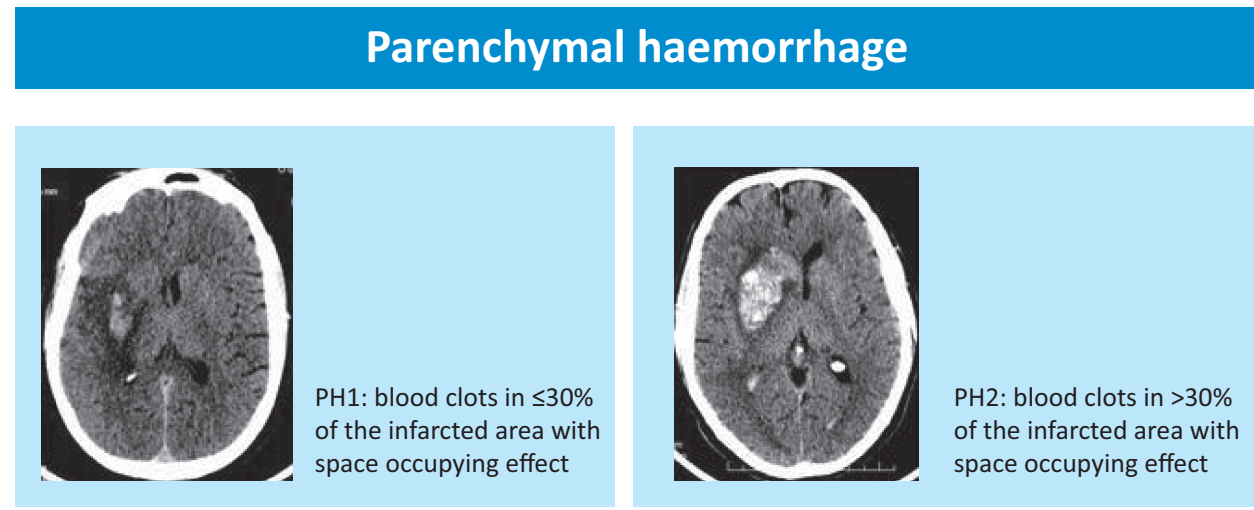

neurovascular services that achieved initiation of IV rtPA within 1-2 hours after onset and reperfusion was achieved in a median of 4-6 hours after onset. Symptom onset to reperfusion times were strongly related to chances of favourable functional outcome, with loss of significant treatment effect by 6 hours in the MR CLEAN trial. ${ }^{24}$ Despite nominally longer time windows for inclusion in some trials ( 8 and 12 hours in REVASCAT and ESCAPE, respectively), very few patients were randomised beyond 6 hours. There was no evidence of different treatment effect by clinical severity, age group, eligibility or ineligibility for IV rtPA, or vessel occlusion site. There were very few data on those with extensive early CT abnormalities or pre-existing disability. Vascular access needs to be feasible and CT angiography should include extra-cranial vessel imaging to determine feasibility of access to the target occlusion.

Complications of endovascular procedures include those related to vascular access, radiological contrast media and device-related vascular injury. Use of general anaesthesia was associated with less favourable outcome compared with sedation in a non-randomised, post-hoc comparison and further trials are in progress to address this question. ${ }^{25}$ Puncture site complications, such as hematoma or haemorrhage, can occur. ${ }^{26}$ Relevant procedural complications reported in the MR CLEAN trial included 5.2\% excess incidence of new ischemic stroke in a different vascular territory, embolisation into new territories outside the target downstream territory of the occluded vessel in $8.6 \%$, procedure-related vessel dissections in $1.7 \%$ and vessel perforations leading to subarachnoid or intracerebral haemorrhage in $0.9 \% .^{27,28}$ Overall, there was no excess of SICH in any of the recent IAT trials. The complication rates quoted in clinical trials reflect practice at expert neurovascular centres, undertaken by interventional neuro-radiologists in almost all cases; whether the same complication rates will pertain to other operators or service configurations is not known.

Incorporating mechanical thrombectomy in routine stroke care is logistically challenging. The number of patients eligible for therapy is likely to be $10-15 \%$ of rtPA treated patients. Centres and individual operators that perform IAT need to treat sufficient cases to maintain efficacy and quality of service. ${ }^{29}$ Clinical and imaging criteria that should prompt patient transfer to a neuro-interventional stroke centre will vary with population density and regional distribution of facilities and expertise. ${ }^{30}$

\section{Conclusions}

Reperfusion by intravenous thrombolysis or endovascular mechanical thrombectomy improves functional outcomes after stroke, but benefit for both treatment modalities is highly time-dependent. Maximum benefit requires minimisation of onset-to-treatment times. The safety and efficacy of IV rtPA is established across a broad range of clinical scenarios. Endovascular treatment now offers greatly improved outcome among patients with poor response to IV rtPA but efficacy has been established only in the context of highly organised neurovascular interventional services.

\section{Conflicts of interest}

KWM is the chief investigator of the Pragmatic Ischaemic Thrombectomy Evaluation (PISTE) trial, funded by the Stroke Association and the 
National Institute of Health Research Health Technology Assessment programme, with unrestricted institutional grants from Codman and Medtronic to support the trial; and received an honorarium for speaking at an advisory board from Boehringer Ingelheim in 2015.

\section{References}

1 Emberson J, Lees KR, Lyden P et al. Effect of treatment delay, age, and stroke severity on the effects of intravenous thrombolysis with alteplase for acute ischaemic stroke: a meta-analysis of individual patient data from randomised trials. Lancet 2014;384:1929-35.

2 Rother J, Ford GA, Thijs VN. Thrombolytics in acute ischaemic stroke: historical perspective and future opportunities. Cerebrovasc Dis 2013;35:313-9.

3 Morris DL, Rosamond W, Madden K, Schultz C, Hamilton S. Prehospital and emergency department delays after acute stroke: the Genentech Stroke Presentation Survey. Stroke 2000;31:2585-90.

4 Frank B, Grotta JC, Alexandrov AV et al. Thrombolysis in stroke despite contraindications or warnings? Stroke 2013;44:727-33.

5 Whiteley WN, Emberson J, Lees KR et al. Risk of intracerebral haemorrhage with alteplase after acute ischaemic stroke: a secondary analysis of an individual patient data meta-analysis. Lancet Neurol 2016;15:925-33.

6 Demaerschalk BM, Kleindorfer DO, Adeoye OM et al. Scientific rationale for the inclusion and exclusion criteria for intravenous alteplase in acute ischemic stroke: a statement for healthcare professionals from the American Heart Association/American Stroke Association. Stroke 2016;47:581-641.

7 Sharma S, Mazya MV, Wahlgren N, Ahmed N. IV thrombolysis in very severe and severe ischemic stroke: results from the SITS-ISTR Registry. Neurology 2016;86:2115.

8 Demchuk AM, Hill MD, Barber PA et al. Importance of early ischemic computed tomography changes using ASPECTS in NINDS rtPA Stroke Study. Stroke 2005;36: 2110-5.

9 Lindley RI, Wardlaw JM, Whiteley WN et al. Alteplase for acute ischemic stroke: outcomes by clinically important subgroups in the Third International Stroke Trial. Stroke 2015;46:746-56.

10 Liebeskind DS, Alexandrov AV. Advanced multimodal CT/ MRI approaches to hyperacute stroke diagnosis, treatment, and monitoring. Ann N Y Acad Sci 2012;1268:1-7.

11 Cheripelli BK, Huang X, MacIsaac R, Muir KW. Interaction of recanalization, intracerebral hemorrhage, and cerebral edema after intravenous thrombolysis. Stroke 2016;47:1761-7.

12 Latchaw RE, Alberts MJ, Lev MH et al. Recommendations for imaging of acute ischemic stroke: a scientific statement from the American Heart Association. Stroke 2009;40:3646-78.

13 European Stroke Organisation (ESO)Executive Committee, ESO Writing Committee. Guidelines for management of ischaemic stroke and transient ischaemic attack 2008. Cerebrovasc Dis 2008;25:457-507.

14 Thomalla G, Sobesky J, Kohrmann M et al. Two tales: hemorrhagic transformation but not parenchymal hemorrhage after thrombolysis is related to severity and duration of ischemia: MRI study of acute stroke patients treated with intravenous tissue plasminogen activator within 6 hours. Stroke 2007;38:313-8.

15 Lansberg MG, Albers GW, Wijman CA. Symptomatic intracerebral hemorrhage following thrombolytic therapy for acute ischemic stroke: a review of the risk factors. Cerebrovasc Dis 2007;24:1-10.
16 Mazya M, Egido JA, Ford GA et al. Predicting the risk of symptomatic intracerebral hemorrhage in ischemic stroke treated with intravenous alteplase: safe Implementation of Treatments in Stroke (SITS) symptomatic intracerebral hemorrhage risk score. Stroke 2012;43:1524-31.

17 Whiteley WN, Emberson J, Lees KR et al. Risk of intracerebral haemorrhage with alteplase after acute ischaemic stroke: a secondary analysis of an individual patient data meta-analysis. Lancet Neurol 2016;15:925-33.

18 Miller DJ, Simpson JR, Silver B. Safety of thrombolysis in acute ischemic stroke: a review of complications, risk factors, and newer technologies. Neurohospitalist 2011;1:138-47.

19 Hurford R, Rezvani S, Kreimei M et al. Incidence, predictors and clinical characteristics of orolingual angio-oedema complicating thrombolysis with tissue plasminogen activator for ischaemic stroke. J Neurol Neurosurg Psychiatry 2015;86:520-3.

20 Zarar A, Khan AA, Adil MM, Qureshi AI. Anaphylactic shock associated with intravenous thrombolytics. Am J Emerg Med 2014;32:113.e3-5.

21 The IST-3 collaborative group. The benefits and harms of intravenous thrombolysis with recombinant tissue plasminogen activator within $6 \mathrm{~h}$ of acute ischaemic stroke (the third international stroke trial [IST-3]): a randomised controlled trial. Lancet 2012;379:2352-63.

22 del Zoppo GJ, Poeck K, Pessin MS et al. Recombinant tissue plasminogen activator in acute thrombotic and embolic stroke. Ann Neurol 1992;32:78-86.

23 Bush CK, Kurimella D, Cross LJ et al. Endovascular treatment with stent-retriever devices for acute ischemic stroke: a meta-analysis of randomized controlled trials. PloS One 2016;11:e0147287.

24 Fransen PS, Berkhemer OA, Lingsma HF et al. Time to reperfusion and treatment effect for acute ischemic stroke: a randomized clinical trial. JAMA Neurol 2016;73:190-6.

25 Berkhemer OA, van den Berg LA, Fransen PS et al. The effect of anesthetic management during intra-arterial therapy for acute stroke in MR CLEAN. Neurology 2016;87:656-64.

26 Darkhabani Z, Nguyen T, Lazzaro MA et al. Complications of endovascular therapy for acute ischemic stroke and proposed management approach. Neurology 2012;79:S192-8.

27 Goyal M, Demchuk AM, Menon BK, et al Randomized assessment of rapid endovascular treatment of ischemic stroke. $N$ Engl J Med 2015;372:1019-30.

28 Berkhemer OA, Fransen PS, Beumer D et al. A randomized trial of intraarterial treatment for acute ischemic stroke. N Engl J Med 2015;372:11-20.

29 Goyal M, Demchuk AM, Hill MD. Endovascular therapy for ischemic stroke. N Engl J Med 2015;372:2366.

30 Fiehler J, Cognard C, Gallitelli M et al. European Recommendations on Organisation of Interventional Care in Acute Stroke (EROICAS). Int J Stroke 2016;11:701-16.

Address for correspondence: Professor Keith W Muir, Institute of Neuroscience and Psychology, University of Glasgow, Queen Elizabeth University Hospital, Glasgow G51 4TF, UK. Email: keith.muir@glasgow.ac.uk 